\title{
Addressing Avoidable Healthcare Costs: Time to Cool Off on Hotspotting in Primary Care?
}

\author{
Leah M. Marcotte, MD ${ }^{7}$, Ashok Reddy, MD, MSc ${ }^{1,2}$, and Joshua Liao, MD, MSc ${ }^{1,3}$ \\ 'Department of Medicine, University of Washington School of Medicine, Seattle, WA, USA; ${ }^{2}$ VA Puget Sound Healthcare System, Seattle, WA, USA; \\ ${ }^{3}$ Leonard Davis Institute of Health Economics, University of Pennsylvania, Philadelphia, PA, USA.
}

One increasingly popular strategy for addressing avoidable healthcare costs is to couple "hotspotting" with interventions that deliver expanded, more intense primary care services to high-cost patient populations. While there is rationale for such intensive primary care programs, early results have been lackluster. Geoffrey Rose's preventive medicine strategy provides insight about a potential explanation: that the narrow scope of these initiatives on small groups of high-cost patients may inherently prevent them from achieving overall cost reductions across entire patient populations. While additional work and results from innovative non-healthcare-based interventions are needed, healthcare organizations may benefit from instead investing in broader interventions that impact patients across cost levels, including average- or low-cost patients.

KEY WORDS: hotspotting; primary care; health economics; preventive medicine.

J Gen Intern Med 34(11):2634-6

DOI: $10.1007 / \mathrm{s} 11606-019-05285-\mathrm{Z}$

(c) Society of General Internal Medicine 2019

$\mathrm{O}$ ver the past decade, a growing number of provider organizations have begun to address healthcare spending in their patient populations through initiatives driven by "hotspotting" - efforts to predict, identify, and address the needs of high-cost patients at the greatest risk for frequent or expensive healthcare utilization.

In primary care, this trend has led to interventions that consist of expanded healthcare services (e.g., intensive case management via multidisciplinary teams) targeted to high-risk patients with complex clinical and social needs. These intensive primary care interventions are based on the premise that because a small segment of the clinical population is responsible for an outsized proportion of avoidable costs, redesigning care delivery for these patients can create outsized cost reductions.

Though intuitive, intensive primary care programs have not consistently reduced costs among high-risk patients. For

Received November 30, 2018

Revised May 30, 2019

Accepted July 24, 2019

Published online August 26, 2019 example, in a randomized control trial of the Intensive Management program (ImPACT) within the Veterans Health Administration (VHA), intensive primary care did not reduce costs or utilization compared with standard primary care. ${ }^{1}$ Moreover, a recent systematic review summarized the impact of intensive primary care interventions for high-risk patients. Despite variation in the structure and design, which ranged from primary care replacement (e.g., home-based) to augmentation (e.g., adding an interdisciplinary team to support primary care), intensive programs as a group have not been clearly associated with improvements in clinical outcomes such as mortality or utilization outcomes such as emergency department or hospital use. ${ }^{2}$

These lackluster results are likely driven by several factors. It can be difficult to intervene on high-cost patients over time given significant turnover. In fact, many individuals identified as high cost in a given year do not achieve the same designation even just one year later. Additionally, intensive primary care programs are also generally focused on amplifying and augmenting traditional primary care services (e.g., chronic disease management) rather than finding novel ways to directly address social determinants of health outcomes and spending (e.g., housing, food insecurity). Another factor is that although different programs can be grouped under the heading of intensive primary care, specific initiatives can vary substantially in structure and strategy. ${ }^{2}$ This heterogeneity can limit the ability to expand or generalize strategies across different primary care populations and organizational settings. Finally, it may be hard to identify improvement targets when not all utilization is avoidable or inappropriate. For example, failing to recognize that many readmissions may not be preventable ${ }^{3}$ could lead to distorted views about how to evaluate a program aimed at reducing them.

\section{INSIGHTS FROM PREVENTIVE MEDICINE THEORY}

Beyond these factors, Geoffrey Rose's preventive medicine strategy provides another potential reason for disappointing results from existing intensive primary care programs. In particular, a key insight from the strategy - which posits that "a large number of people exposed to a small risk may generate many more cases than a small number exposed to a high

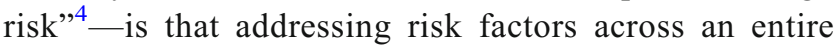


population can produce greater impact on outcomes than emphasis on a small group of high-risk individuals.

Consider an example of efforts to reduce unplanned hospitalizations. ${ }^{5}$ Because most of the individuals in a population are average risk (e.g., $80 \%$ of the population) rather than high risk (e.g., $0.5 \%$ of the population), the former will account for the majority of admissions. In turn, one would have to achieve far greater admission reductions among high-risk versus averagerisk individuals to produce the same overall effects (e.g., achieving an overall 5\% reduction in admissions would require a 54\% decrease in admissions among high-risk individuals, compared with a $13 \%$ decrease among average-risk individuals).

These insights pose two important implications for provider organizations attempting to address avoidable healthcare costs in primary care. First, although high-cost patients may demonstrate more utilization than others on an individual basis, the cumulative costs for larger groups of average- or low-cost individuals as a group are also considerable. Therefore, interventions that focus on the latter can be easier to execute while generating meaningful reductions in overall spending.

For example, analyses of Medicare patients demonstrate that $17 \%$ highest cost individuals have almost twice the rate of lowvalue services as the rest of the population. However, despite the concentration of inappropriate spending among this highcost group, it only accounts for $27 \%$ of total low-value services. ${ }^{6}$ Instead, the vast majority of the low-value care services (73\%) occur among the larger group of non-high-cost patients. In turn, interventions focused disproportionately on high-cost patients may have only limited impact on reducing low-value services at the overall population level by leaving cost drivers among average- or low-cost patients unaddressed.

Second, compared with hotspotting-driven intensive primary care interventions focused on high-cost patients, initiatives targeting utilization across a broader range of individuals may be more cost-efficient and widely applicable. For example, teams in the ImPACT program, which included a physician, nurse practitioner, social worker, and recreation therapist, were responsible for providing healthcare services to small panels of 150 patients. In contrast, as a broader intervention in the same system, the VHA patient-centered medical home model uses similarly constructed teams (consisting of a physician, nurse, and administrative support staff) to deliver primary care services to nearly 1200 patients while matrixing part-time social workers, mental health, and pharmacist support across multiple patient panels. Though the costs for broader population-based interventions may be higher in the absolute sense due to the larger number of patients, these initiatives may be more cost-efficient on a per-patient basis. Regardless, pilot tests could also be conducted to evaluate local cost feasibility before full implementation.

Moreover, broader interventions can address utilization among high-cost patients without being specifically targeted to them, leading to wider applicability and impact. For example, because they share some of the same utilization drivers, high- and low-cost patients may both benefit from interventions that attempt to expand access to primary care services in order to decrease emergency department utilization. Such initiatives would be agnostic to individuals' cost and risk levels, spreading financial overhead across a larger group of patients. Adopting this type of approach in its patientcentered medical home model, the VHA expanded same day primary care access for patients across all risk levels. In this model, patients at clinics that delivered the highest degree of team-based care had fewer emergency department visits and hospitalizations for ambulatory sensitive conditions. ${ }^{7}$

\section{FUTURE DIRECTIONS}

Given the persistent challenge of avoidable healthcare costs, organizations should continue to evaluate the benefits of hotspotting-driven interventions focused on high-cost patients. We believe that high-cost patients can require complex care that often goes beyond the walls of the primary care clinic. In particular, interventions that extend beyond healthcare into the realms of public health and social services may hold promise ${ }^{8}$ and provide insight about the role of nontraditional interventions in addressing avoidable healthcare costs. Additionally, we acknowledge that there is room for more research about intensive primary care models, particularly in different settings including those beyond the VHA. In that vein, we look forward to findings from an ongoing study of intensive primary care and the insight it will provide about the potential role of hotspotting-based primary care strategies. ${ }^{9}$

Nonetheless, while we await the results from such efforts, existing evidence raises questions about whether organizations can consistently and materially curb avoidable costs by providing more intense or expanded primary care services to small, high-cost populations. Insights from Rose's preventive medicine strategy suggest that one potential solution is to invest time and resources in interventions targeted at individuals across a clinical population.

Ultimately, these concepts underscore the inherent tradeoffs facing clinicians and healthcare organizations tasked with addressing inappropriate costs with limited organizational resources. As one example, the resource requirements of intensive primary care programs could restrict providers' ability to care for other large segments of the patient population. For example, most provider organizations - where average primary care panels consist of 2000 or more patients - would be strained by team structures focused on several hundred patients only, such as those used in the ImPACT program. Such staffing challenges would be further exacerbated by the existing shortage of primary care providers.

\section{CONCLUSION}

Multiple strategies are likely needed to address the persistent challenge of avoidable healthcare costs. However, organizations must make investment and programming trade-offs 
regarding not just which programs to adopt, but which ones to modify or de-adopt. Existing evidence and principles from preventive medicine suggest that on their own, hotspottingdriven intensive primary care interventions may be unlikely to "solve" the problem. As we await results from intensive primary care programs implemented in different settings, as well as programs that use hotspotting to deliver non-traditional interventions to high-cost patients, healthcare organizations should consider investing in interventions that impact patients across cost and risk levels.

Corresponding Author: Leah M. Marcotte, MD; Department of Medicine University of Washington School of Medicine, Seattle, WA, USA (e-mail:leahmar@uw.edu).

\section{Compliance with Ethical Standards:}

Conflict of Interest: Dr. Liao reports textbook royalties from Wolters Kluwer and personal fees from Kaiser Permanente Washington Research Institute, none of which are related to this manuscript. Dr. Marcotte and Dr. Reddy declare that they do not have a conflict of interest.

\section{REFERENCES}

1. Zulman D, et al. Effect of an Intensive Outpatient Program to Augment Primary Care for High-Need Veterans Affairs Patients: A Randomized Clinical Trial. JAMA Intern Med. 2017;177:166-175.
2. Edwards ST, Peterson K, Chan B, Anderson J, Helfand M. Effectiveness of Intensive Primary Care Interventions: A Systematic Review. J Gen Intern Med. 2017;32:1377-1386

3. van Walraven C, Bennett C, Jennings A, Austin PC, Forster AJ. Proportion of hospital readmissions deemed avoidable: a systematic review. CMAJ. 2011;183:E391-E402

4. Rose GA, Khaw K-T, Marmot MG. Rose's Strategy of Preventive Medicine: The Complete Original Text. Oxford: Oxford University Press; 2008. Print.

5. Martin R, Gary A. Reducing emergency admissions: are we on the right track? BMJ. 2012;345:e6017.

6. McWilliams JM, Schwartz AL. Focusing on High-Cost Patients - The Key to Addressing High Costs? NEJM. 2017;376:807-809.

7. Nelson $\mathbf{K}$, et al. Implementation of the Patient-Centered Medical Home in the Veterans Health Administration: Associations with Patient Satisfaction, Quality of Care, Staff Burnout, and Hospital and Emergency Department Use. JAMA Int Med. 2014;174:1350-1358.

8. Larimer ME, Malone DK, Garner MD, et al. Health Care and Public Service Use and Costs Before and After Provision of Housing for Chronically Homeless Persons With Severe Alcohol Problems. JAMA. 2009;301(13):1349-1357.

9. ClinicalTrials.gov. Identifier: NCT02090426. "Health Care Hotspotting: A Randomized Controlled Trial." Accessed 20 August 2019. Available: https: / / clinicaltrials.gov/ct2/show/study/NCT02090426?show_ desc $=$ Y\#desc.

Publisher's Note Springer Nature remains neutral with regard tojurisdictional claims in published maps and institutional affiliations. 